\title{
Determination of the elastic modulus of ascending thoracic aortic aneurysm at different ranges of pressure using uniaxial tensile testing
}

\author{
Khalil Khanafer, $\mathrm{PhD},{ }^{\text {a,b }}$ Ambroise Duprey, $\mathrm{MD},{ }^{\mathrm{a}, \mathrm{c}}$ Mohammad Zainal, $\mathrm{PhD},{ }^{\mathrm{d}}$ Marty Schlicht, $\mathrm{PhD},{ }^{\mathrm{a}}$ \\ David Williams, MD, ${ }^{\mathrm{e}}$ and Ramon Berguer, $\mathrm{PhD}, \mathrm{MD}^{\mathrm{a}, \mathrm{b}}$
}

\begin{abstract}
Objective: The purpose of this study is to provide measurements of the elastic modulus of the aortic wall of ascending thoracic aortic aneurysms for different ranges of pressure (physiologic, hypertensive). In addition, pre-failure stress, taken as the peak stress obtained before specimen failure, was recorded for each test.
\end{abstract}

\begin{abstract}
Methods: Ninety-seven aortic samples freshly excised from 13 patients with ascending thoracic aortic aneurysms were obtained from greater and lesser curvatures and tested uniaxially in circumferential and longitudinal orientations.
\end{abstract}

\begin{abstract}
Results: The maximum elastic moduli, overall, and particularly in the lesser curvature were significantly higher in the circumferential orientation (9.19 MPa) than in the longitudinal (3.13 MPa). Results of peak stress showed positive correlation with maximum elastic modulus and inverse correlation with tissue wall thickness.
\end{abstract}

Conclusions: This study provides new data on the elastic modulus in the physiologic and hypertensive range that can be used in computational analysis and the design of bench-top models. The accuracy of computational analysis and bench-top models strongly depends on the knowledge of the elastic properties of the aortic wall. The mechanical properties presented in this study, with specific values for 2 locations (greater and lesser curvature) and 2 directions (circumferential, longitudinal), will increase our understanding of the mechanisms that precede rupture of an ascending aortic aneurysm. (J Thorac Cardiovasc Surg 2011;142:682-6)

Cardiovascular disease is the leading cause of death in the modern world. Ascending thoracic aortic aneurysms (ATAAs) cause death by rupture. The estimated incidence of thoracic aortic aneurysms is 6 cases per 100,000 persons in the United States every year. ${ }^{1,2}$ The diagnosis of aortic aneurysms has increased significantly in the last 30 years because of an increase in the use of imaging. Fifty percent of patients with a ruptured thoracic aortic aneurysm die before reaching the hospital. Furthermore, surgical repair of a ruptured thoracic aneurysm carries $25 \%$ to $50 \%$ mortality as opposed to $5 \%$ to $8 \%$ mortality when such aneurysms are treated electively. The decision to intervene is currently based on the maximum diameter. An ATAA with a maximum diameter between 5.5 and $6.0 \mathrm{~cm}$ is considered at high risk for rupture and usually recommended for surgical repair. The computational tools available today

\footnotetext{
From the Vascular Mechanics Laboratory, ${ }^{a}$ Department of Biomedical Engineering, and Section of Vascular Surgery, ${ }^{\mathrm{b}}$ University of Michigan, Ann Arbor, Mich; Ecole Nationale Supérieure des Mines, ${ }^{c}$ St Etienne, France; Department of Quantitative Methods and Information Systems College of Business Administration, ${ }^{\mathrm{d}}$ Safat, Kuwait; and Department of Radiology, ${ }^{\mathrm{e}}$ University of Michigan, Ann Arbor, Mich. This work was supported by the Frankel Vascular Research Fund.

Disclosures: Authors have nothing to disclose with regard to commercial support.

Received for publication Feb 18, 2010; revisions received Aug 11, 2010; accepted for publication Sept 26, 2010; available ahead of print May 26, 2011.

Address for reprints: Khalil Khanafer, $\mathrm{PhD}$, Biomedical Engineering Department, University of Michigan, Ann Arbor, MI 48109 (E-mail: khanafer@umich.edu). 0022-5223/\$36.00

Copyright (C) 2011 by The American Association for Thoracic Surgery doi:10.1016/j.jtcvs.2010.09.068
}

permit the calculation of stresses in the aneurysmal wall and offer a more accurate tool to assess the risk of rupture of an ATAA than the measurement of its diameter. However, the ability to perform accurate computations of the stresses bearing on the wall of an aneurysm requires precise knowledge of its mechanical properties.

The biomechanical properties of ATAAs have been studied. $^{2-11}$ Vorp and colleagues ${ }^{8}$ determined the tensile strength and maximum tissue stiffness of ATAA tissues in a uniaxial tensile testing machine. Tissue strips of longitudinal or circumferential orientations were stretched to failure. They showed that the tensile strength of an ATAA was less than that of normal aortic specimens, whereas the maximum elastic modulus (MEM) of an ATAA was greater (stiffer) than that of tissue controls. They did not find a significant difference in MEM between circumferential and longitudinal specimens for either aneurysmal or control tissue. Iliopoulos and colleagues ${ }^{10}$ studied the regional and directional differences in the mechanical properties of ATAAs using uniaxial tensile testing. They did not find a correlation among tissue strength, ATAA diameter, and patient age. They found higher stiffness in the circumferential than longitudinal orientation. Koullias and colleagues ${ }^{11}$ measured the mechanical properties of the aorta by direct epiaortic echocardiography in patients undergoing ascending aortic aneurysm replacement and in 20 control patients with normal aortas undergoing coronary artery bypass grafting. They found that the mechanical properties of the aneurysmal aorta deteriorate dramatically as the aorta enlarges, 


\section{Abbreviations and Acronyms}

ATAA $=$ ascending thoracic aortic aneurysm

MEM $=$ maximum elastic modulus

reaching critical levels associated with rupture by a diameter of $6 \mathrm{~cm}$.

On review of the existing data on the mechanical properties measurements of aneurysms, we encountered the following methodological problems. First, different stress-strain curve definitions have been used in the literature to determine the elasticity of the wall of aneurysms. ${ }^{12-17}$ Vorp and colleagues ${ }^{8}$ and other authors ${ }^{13,14}$ determined the mechanical properties of aortic aneurysms by plotting the stress-strain curve using true stress versus engineering strain. Their choice of this method remains unclear to us. Other researchers have used the engineering stress-engineering strain definition. ${ }^{9,15,16}$ But this method is based on the original dimensions of the specimen being tested and thus is not applicable to the tensile testing of aortic strips that, during testing, undergo a larger deformation than is observed in living aortas. We propose that the true stress-true strain relationship is the best method to describe the stress-strain relationship in arteries because it takes into account the changes of dimensions of the specimen during tensile testing. We used this method in the present study under the assumption of the incompressibility of the tissue. ${ }^{18}$

The second issue is associated with determining the mechanical properties of an ATAA. The studies in the literature have focused on the determination of the MEM and the tensile strength of the ATAA. None of the published studies measured the different elastic behavior of the ATAA walls at the physiologic and hypertensive ranges of pressure.

We determined the mechanical properties of the ATAA wall at 3 points using aortic wall strips obtained from the greater and lesser curvatures in circumferential and longitudinal orientations. They were tested at (1) physiologic pressure range $(80-120 \mathrm{~mm} \mathrm{Hg}$ ), (2) hypertensive range (systolic $\geq 160 \mathrm{~mm} \mathrm{Hg}$, diastolic $\geq 100 \mathrm{~mm}$ $\mathrm{Hg}$ ), and (3) from the maximum slope of the stress-strain curve. In addition, pre-failure stress taken as the peak stress obtained before specimen failure was recorded for each test.

\section{MATERIALS AND METHODS}

\section{Human Aortic Tissue Specimens}

All procedures were carried out in accordance with the guidelines of the institutional review board of the University of Michigan. Segments of aortic wall were excised from surgical specimen obtained from patients undergoing surgical repair of ATAAs. These segments were cut into $3 \times 30$-mm strips of circumferential and longitudinal orientation with custom-designed tissue cutters. Samples were obtained from the greater and lesser curvatures. The tissue specimens were stored in gauze wetted with saline and refrigerated at $4^{\circ} \mathrm{C}$. Tissue testing was performed within 48 hours. After equilibration at room temperature, adipose tissue was removed from the surface of the adventitia and the $3 \times 30$ - $\mathrm{mm}$ strips (both circumferential and longitudinal orientations) were tested uniaxially. Original thickness and width of the sample were measured at the zero stress state with a digital caliper. Multiple measurements were used, with their averages being used in the subsequent calculations of the elastic modulus.

\section{Uniaxial Tensile Testing}

Experiments were carried out in a tensile testing machine (Instron model 5542; Instron Inc, Norwood, Mass). Adhesive sandpaper of fine grit was placed on the surface of the jaws of the pneumatic grips to prevent slippage. During preliminary testing, we found that an aspect ratio of at least 2 was required to ensure a free shear deformation and local narrowing of the tissue between the grips. The tissue was continuously wetted with phosphate-buffered saline before and during the mechanical testing. As is advisable when testing viscoelastic soft tissues, each specimen was preconditioned by executing 10 cycles at a constant crosshead speed $(10 \mathrm{~mm} /$ min) to obtain repeatable stress-strain curves. Thereafter, the sample underwent the uniaxial extension test with continuous recording of tensile force and extension at the same crosshead speed until failure.

\section{Data Analysis}

The load-stretch curve was derived to obtain the stress-strain relationship. We assumed the aortic wall to be an incompressible material ${ }^{18}$ and calculated the true stress and true strain relationship. To calculate the true stress and true strain, we need to define engineering stress and strain, which depend on the initial dimensions of the tissue. The engineering stress is

$$
\sigma_{E}=\frac{F}{A_{o}}
$$

where $F$ is the load and $A_{o}$ is the initial cross-sectional area. The engineering strain is

$$
\epsilon_{E}=\frac{\Delta L}{L_{o}}
$$

where $\Delta L$ is the change in length and $L_{o}$ the initial length. Then we define the true stress as

$$
\sigma_{T}=\frac{F}{A}
$$

where $A$ is the current cross-sectional area. The true strain is defined by

$$
d \epsilon_{T}=\frac{d L}{L}
$$

where $d L$ is the instantaneous stretch and $L$ the current length of the specimen. The assumption of the arterial incompressibility implies a zero change of volume during the tensile testing: $A \times L=A_{o} \times L_{o}$ and then

$$
\sigma_{T}=\frac{F}{A}=\frac{F}{A_{o}} \frac{L}{L_{o}}=\sigma_{E} \times \frac{L_{o}+\Delta L}{L_{o}}=\sigma_{E}\left(1+\epsilon_{E}\right)
$$

The true strain is defined as the sum of all the current engineering strains, and then

$$
\epsilon_{T}=\int d \epsilon=\int_{L_{o}}^{L} \frac{d L}{L}=\ln \frac{L}{L_{o}}=\ln \frac{L_{o}+\Delta L}{L_{o}}=\ln \left(1+\epsilon_{E}\right)
$$


To characterize the physiologic and hypertensive moduli of the specimen, the Laplace law was used to obtain the circumferential stress $\sigma_{\theta}$, assuming the aneurysm to be of nearly cylindric shape:

$$
\sigma_{\theta}=\frac{P R}{t}
$$

where $P$ is the pressure, $R$ is the radius of the aneurysm (obtained from the computed tomography angiogram of the patient at $75 \%$ of the cardiac cycle), and $t$ is the thickness of the specimen. Pressure ranges of 80 to $120 \mathrm{~mm}$ $\mathrm{Hg}$ and 100 to $240 \mathrm{~mm} \mathrm{Hg}$ were designated as the physiologic and hypertensive ranges, respectively (Figure 1). Because the stress-strain curve is nearly linear within this short range, a linear fitting was applied yielding the physiologic and hypertensive elastic modulus calculated as the slope of the fitted line.

\section{Statistics}

Data processing and statistical analysis were performed using Minitab software (Minitab Inc, State College, Pa). The present investigation consists of 4 pertinent dependent variables (physiologic modulus, hypertensive modulus, maximum modulus, and peak stress) and 2 factors (location and direction each with 2 levels). Greater and lesser curvatures are the 2 location levels, and circumferential and longitudinal are the 2 directional levels. At first, a general linear model was used to check if there was any interaction effect between direction and location (ie, location effect is modified by changing direction or vice versa). Application of a general linear model showed that the interaction effect of direction and location was statistically insignificant. Therefore, one-at-a-time factor tests were conducted for separate location (greater and lesser curvatures) and direction (circumferential and longitudinal). Descriptive statistics (mean \pm standard deviation) were calculated for each set of data. Multiple specimens from individual patients were separately analyzed, and the elastic modulus values were averaged to yield one value per study subject. Student $t$ test was performed to assess the influence of location and orientation on the elastic modulus for various pressure ranges.

\section{RESULTS}

Ninety-seven specimens obtained from 13 patients (aged 39-75 years) with ATAAs (54.2 $\pm 7.32 \mathrm{~mm}$; mean \pm standard deviation) provided 68 circumferential-oriented specimens (greater curvature, 42; lesser curvature, 26) and 29 longitudinal-oriented specimens (greater curvature, 16; lesser curvature, 13) for tensile testing. The results of the testing are shown in Figures 2 to 5. By using a 2-sample $t$ test for

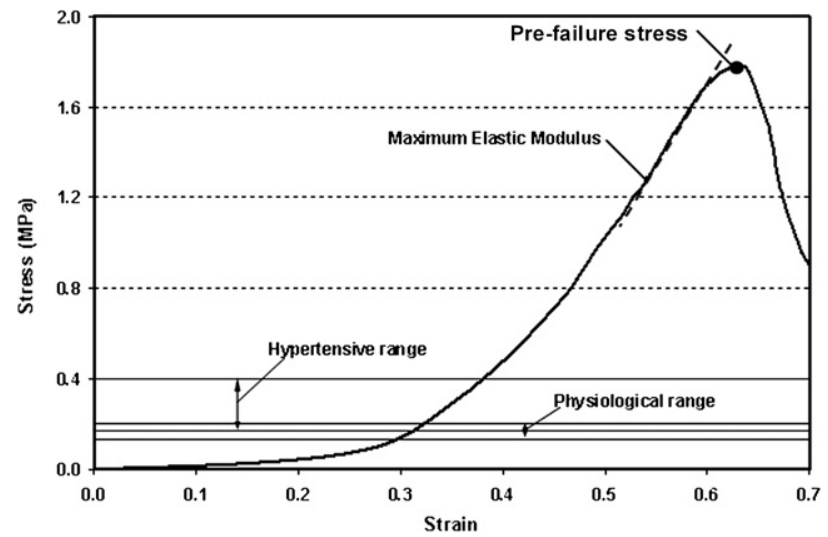

FIGURE 1. Principles of calculating the physiologic range, hypertensive range, and MEM.

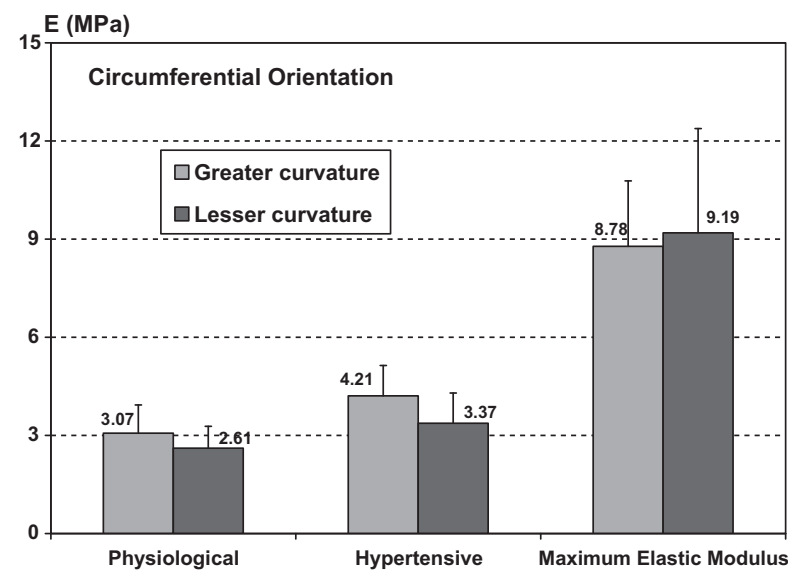

A

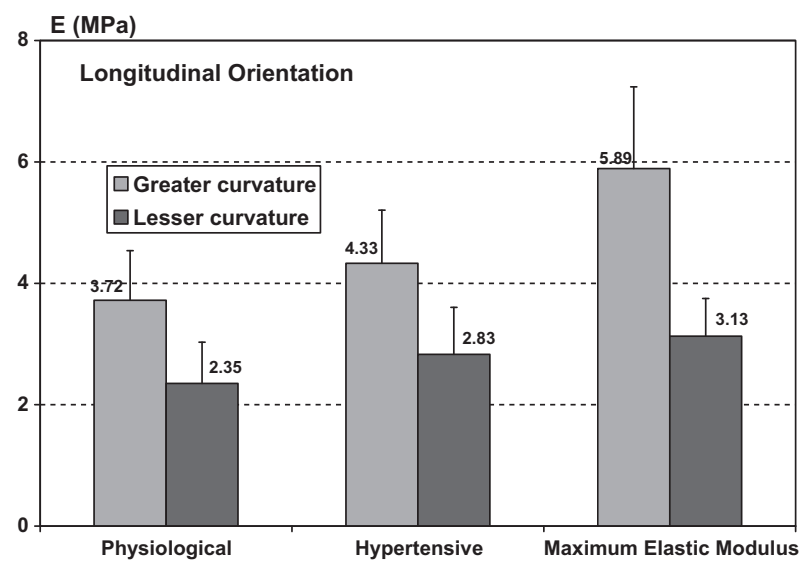

B

FIGURE 2. Comparison of the elastic modulus between greater curvature and lesser curvature locations in the (A) circumferential and (B) longitudinal orientations for various pressure ranges.

the 3 dependent variables (physiologic, hypertensive, and MEM) with respect to changing location, our MEM results indicated no significant difference in the circumferential group between greater and lesser curvature locations

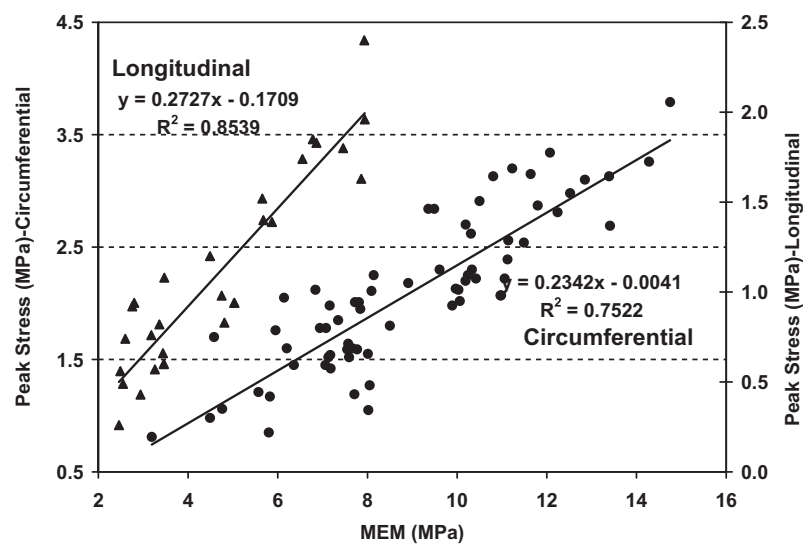

FIGURE 3. Correlation between MEM and peak stress in the circumferential and longitudinal orientations. MEM, Maximum elastic modulus. 


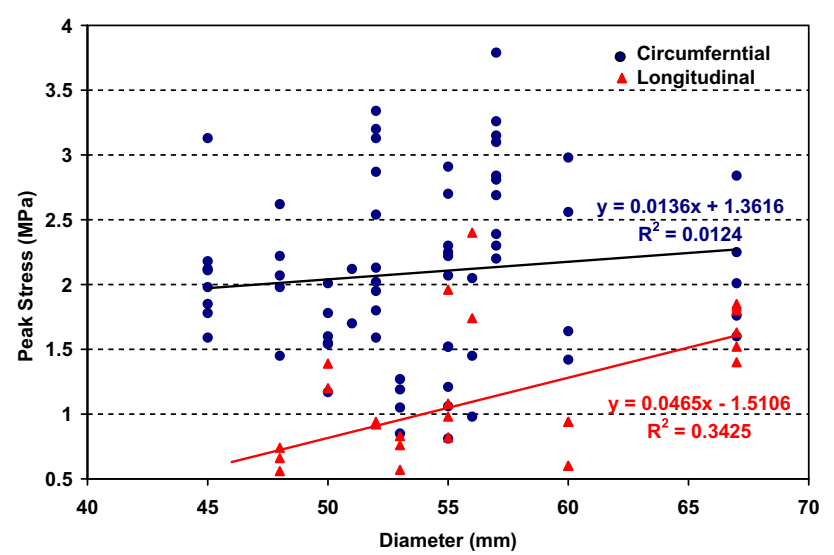

FIGURE 4. Effect of the aneurysm diameter on peak stress in the circumferential and longitudinal orientations.

$(P=.558)$, whereas the difference between greater and lesser curvature locations was significant in the longitudinal group $(P=.000)$, with the greater curvature specimen being stiffer than the lesser curvature specimen (Figure 2). Because of the relatively small number of observations available for the longitudinal group, the Mann-Whitney test was carried out in this group to examine the effect of location instead of the 2 -sample $t$ test. The Mann-Whitney method is a nonparametric test for assessing whether 2 independent samples of observations have equally large values.

In the greater curvature specimen (Figure 2), the MEM was considerably higher in the circumferential strips than in the longitudinal strips $(P=.000)$, whereas no significance was found for the elastic modulus in the hypertensive range $(P=.514)$. In the lesser curvature specimen, the effect of direction on the dependant variables was not found to be significant in the physiologic range $(P=.2514)$ or hypertensive range $(P=.079)$. However, they were significantly different for MEM $(P=.000)$. The effect of patient age on the peak stress (tissue tensile strength) was

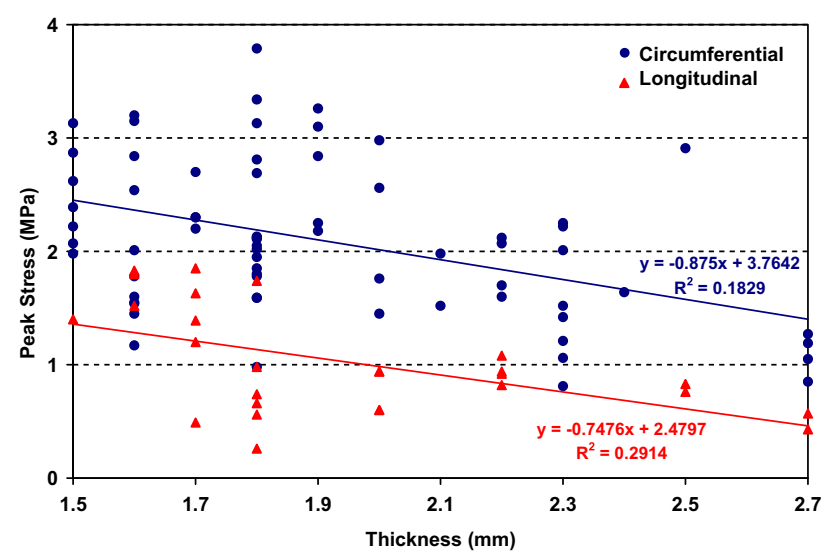

FIGURE 5. Effect of the aneurysm wall thickness on peak stress in the circumferential and longitudinal orientations. studied in this investigation (data not shown). The age of the patient does not correlate with peak stress in either circumferential or longitudinal directions.

\section{DISCUSSION}

The modulus of elasticity is a datum that describes how a material will deform or break when subjected to a force or stress. The initiation, growth, and eventual rupture of an aneurysm are prompted by changes in elasticity of the aortic wall. Knowledge of the elasticity of the aorta is also important for the design of aortic endografts because their stability, once inserted, depends to a large degree on the elastic behavior of the segment of the aorta to which they are attached.

\section{Effect of Location and Orientation on the Elastic Modulus}

The effect of location on MEM values of ATAA was studied by Choudhury and colleagues ${ }^{9}$ : MEM was not significantly stiffer in the greater curvature than lesser curvature in either the longitudinal or the circumferential strips. In our measurements of MEM, the greater curvature is significantly stiffer than the lesser curvature in the longitudinal strips. Also, in the lesser curvature, the MEM was substantially stiffer in the circumferential orientation than in the longitudinal orientation. Thubrikar and colleagues ${ }^{14}$ studied the influence of location on the elastic modulus of abdominal aortic aneurysms. In the longitudinal strips, the lateral and anterior walls showed greater stiffness than the posterior wall. We measured the elastic moduli in the physiologic and hypertensive ranges of pressure at the greater and lesser curvatures. There was a significant difference in the physiologic range $(P=.016)$ and highly significant difference in the hypertensive range $(P=.001)$. The difference between greater and lesser curvatures was also highly significant in the longitudinal specimens $(P=.000)$.

Our results suggest that the formation of an ATAA is associated with directional variations in the mechanical properties of an ATAA, such as weakening and stiffening of the aortic wall. Changes in these mechanical properties alter the likelihood of aneurysmal rupture. Clinical observation shows that most aortic type A dissections occur with a tear at the greater curvature of the ascending aorta. ${ }^{19}$ The main finding of our results is that the greater curvature has a higher elastic modulus than the lesser curvature in the longitudinal direction. From a clinical point of view, this greater curvature exhibits higher stresses than the lesser curvature and is more likely to rupture or dissect.

There are conflicting results in the literature regarding the effect of orientation on the elastic modulus. Vorp and colleagues ${ }^{8}$ showed no significant difference in MEM between circumferential and longitudinal specimens. However, Iliopoulos and colleagues ${ }^{10}$ recently reported significant differences in the failure stress and MEM between the 
circumferential and longitudinal directions in all regions. The MEM was significantly stiffer in the circumferential orientation than in longitudinal orientation. Thubrikar and colleagues ${ }^{14}$ reported that the circumferential strips were stiffer than the longitudinal strips in abdominal aortic aneurysms. Peterson and colleagues ${ }^{3}$ studied ATAA using biaxial testing and reported 1 of 6 patients with stiffer specimens (higher elastic modulus) in the circumferential orientation. We found that the MEM was significantly stiffer in circumferential orientation than in longitudinal orientation. Rupture may well occur transversely because peak stress in the transverse direction is greater than peak stress in the longitudinal direction.

\section{Correlations Among Peak Stress and Maximum Elastic Modulus, Peak Stress and Diameter, and Peak Stress and Thickness}

The tissue peak stress and MEM correlated significantly in the circumferential and longitudinal orientations (Figure 3). However, the correlation between aneurysm diameter and the tissue peak stress was weak in the longitudinal orientation and nonexistent in the circumferential orientation (Figure 4). Figure 5 shows an inverse correlation between peak stress and wall thickness in both orientations.

The role of wall stresses in the development of transverse intimal tears in aortic dissections was previously studied numerically using a finite element method by Thubrikar and colleagues. ${ }^{20}$ These authors concluded that during the aneurysm growth, the increase in the longitudinal stress is the most likely cause of aortic dissection because the peak stress (tensile strength) will be reached first in the longitudinal direction. Our results confirm the fact that the peak stress is lower in the longitudinal direction than in the circumferential direction for a given diameter.

\section{CONCLUSIONS}

By using a true stress-true strain definition, we measured the elastic modulus and MEM of the aortic wall of ATAAs within physiologic and hypertensive ranges of pressure. We produced a wide range of elastic data for more accurate modeling of the stresses acting on ATAAs and for the design of bench-top aortic models. In longitudinal orientation, the MEM was different for the greater and lesser curvatures. The MEM was greater in the circumferential than in the longitudinal orientation. We found a direct correlation between peak stress and MEM and a negative correlation between peak stress and aneurysm wall thickness.

\section{References}

1. Bickerstaff LK, Pairolero PC, Hollier LH, Melton LJ, Van Peenen HJ, Cherry KJ, et al. Thoracic aortic aneurysms: a population-based study. Surgery. 1982;92: 1103-8.

2. Ruddy JM, Jones JA, Spinale FG, Ikonomidis JS. Regional heterogeneity within the aorta: relevance to aneurysm disease. J Thorac Cardiovasc Surg. 2008;136: 1123-30.

3. Peterson SJ, Sundt TM III, Kouchoukos NT, Yin FCP, Okamoto RJ. Biaxial mechanical properties of dilated human ascending aortic tissue. BMES/EMBS Conference. In: Proceedings of the First Joint, Oct 13-16, 1999, Atlanta, GA. $1999 ; 1: 195$.

4. Okamoto RJ, Wagenseil JE, DeLong WR, Peterson SJ, Kouchoukos NT, Sundt TM. Mechanical properties of dilated human ascending aorta. Ann Biomed Eng. 2002;30:624-35.

5. Okamoto RJ, Xu H, Kouchoukos NT, Moon MR, Sundt TM III. The influence of mechanical properties on wall stress and distensibility of the dilated ascending aorta. J Thorac Cardiovasc Surg. 2003;126:842-50.

6. Sundt TM III. Residual strain in the aorta. J Thorac Cardiovasc Surg. 2006;131: 1420-1.

7. Iliopoulos DC, Kritharis EP, Giagini AT, Papadodima SA, Sokolis DP. Ascending thoracic aortic aneurysms are associated with compositional remodeling and vessel stiffening but not weakening in age-matched subjects. $J$ Thorac Cardiovasc Surg. 2009; 137:101-9.

8. Vorp DA, Schiro BJ, Ehrlich MP, Juvonen TS, Ergin MA, Griffith BP. Effect of aneurysm on the tensile strength and biomechanical behavior of the ascending thoracic aorta. Ann Thorac Surg. 2003;75:1210-4.

9. Choudhury N, Bouchot O, Rouleau L, Tremblay D, Cartier R, Butany J, et al. Local mechanical and structural properties of healthy and diseased human ascending aorta tissue. Cardiovasc Pathol. 2009;8:83-91.

10. Iliopoulos DC, Devejab RP, Kritharisc EP, Perreaa D, Sionisa GD, Toutouzasd K, et al. Regional and directional variations in the mechanical properties of ascending thoracic aortic aneurysms. Med Eng Phys. 2009;31:1-9.

11. Koullias G, Modak R, Tranquilli M, Korkolis DP, Barash P, Elefteriades JA. Mechanical deterioration underlies malignant behavior of aneurysmal human ascending aorta. J Thorac Cardiovasc Surg. 2005;130:677-83.

12. Hundía N, Ratcliffe MB, Pruitt LA. Determination of strain energy function for arterial elastin: experiments using histology and mechanical tests. J Biomech. 2007;40:586-94.

13. He CM, Roach MR. The composition and mechanical properties of abdominal aortic aneurysms. J Vasc Surg. 1994;20:6-13.

14. Thubrikar MJ, Labrosse M, Robicsek F, Al-Soudi J, Fowler B. Mechanical properties of abdominal aortic aneurysm wall. J Med Eng Technol. 2001;25:133-42.

15. Raghavan ML, Webster MW, Vorp DA. Ex vivo biomechanical behavior of abdominal aortic aneurysm: assessment using a new mathematical model. Ann Biomed Eng. 1996;24:573-82.

16. Emmanuel MK, Dimitrios PS. Morphometric study of stress-induced changes in aortic wall microstructure. Proceedings of the Fifth IASTED International Conference: Biomedical Engineering. Innsbruck, Austria: ACTA Press; 2007.

17. Sokolis DP. Passive mechanical properties and structure of the aorta: segmental analysis. Acta Physiol (Oxf). 2007;190:277-89.

18. Carew TE, Vaishnav RN, Patel DJ. Compressibility of the arterial wall. Circ Res. 1968;23:61-8

19. Hebballi R. Diagnosis and management of aortic dissection. Continuing Educ Anaesth Crit Care Pain. 2009;9:14-18.

20. Thubrikar MJ, Agali P, Robicsek F. Wall stress as a possible mechanism for the development of transverse intimal tears in aortic dissections. J Med Eng Technol. 1999;23:127-34. 